\title{
GENEALOGY OF PERIODIC POINTS OF MAPS OF THE INTERVAL
}

BY

\author{
ROBERT L. DEVANEY ${ }^{1}$
}

\begin{abstract}
We describe the behavior of families of periodic points in one parameter families of maps of the interval which feature a transition from simple dynamics with finitely many periodic points to chaotic mappings. In particular, we give topological criteria for the appearance and disappearance of these families. Our results apply specifically to quadratic maps of the form $F_{\mu}(x)=\mu x(1-x)$.
\end{abstract}

0. Introduction. In recent years, much attention has been paid to the dynamical properties of smooth mappings of the real line to itself. The logistic function $g_{\mu}(x)=\mu x(1-x)$ in particular has been studied by many authors. As the parameter $\mu$ for this family is varied through positive values, the associated dynamical systems become increasingly more complex. For example, when $0<\mu<1$, there are only two fixed points. All other points tend either to one of these two fixed points or else to $-\infty$ under iteration. As $\mu$ increases further, it can be shown that more and more periodic orbits appear in a regular fashion and that as long as $\mu$ is less than approximately 3.57 , the system remains relatively simple, i.e., there are only finitely many periodic points.

On the other hand, when $\mu>4$, the dynamical system is much more complicated yet still well understood. All points tend to $-\infty$ with the exception of a subset $\Lambda$ of the unit interval which is mapped into itself. $\Lambda$ is known to be homeomorphic to a Cantor set (see $\S 1$ below) and, moreover, the restriction of $g_{\mu}$ to $\Lambda$ is topologically conjugate to a one-sided shift automorphism on two symbols. In particular, there are $2^{n}$ fixed points for $g_{\mu}^{n}$, so the dynamics here are quite a bit different from those for lower values of $\mu$. A major question in bifurcation theory is how does one progress from the simple dynamical systems for $\mu<3.57$ to the more complex systems found for higher values of $\mu$. One of our goals in this paper is to investigate how the infinitely many periodic orbits arise as $\mu$ approaches 4 .

More specifically, we study families of mappings $f_{\mu}$ which resemble the logistic function in that $f_{0}$ is identically 0 and $f_{\mu}$ has an invariant set $\Lambda_{\mu}$ similar to the above when $\mu \geqslant b$. The precise hypotheses are given in $\S 1$. For these mappings we show that a particular point arises via a finite sequence of simple bifurcations. We catalogue these bifurcations in the sense that we present an algorithm that decides which lower period points give rise via a sequence of bifurcations to a given

Received by the editors October 9, 1979 and, in revised form, April 18, 1980.

1980 Mathematics Subject Classification. Primary 58F14, 58F22; Secondary 28D99.

${ }^{1}$ Research partially supported by NSF Grant MCS 78-00430. 
periodic point in $\Lambda_{\mu}$. There may be other nonessential bifurcations along the way, but our result shows that where a given periodic point in $\Lambda_{\mu}$ is "born" is independent of the particular family of mappings. This can be viewed as a reconstruction of the past history of the periodic points.

On the other hand, we also discuss the "future" behavior of a periodic point. That is, we present an algorithm which lists all higher period points which ultimately bifurcate away from the given periodic point as $\mu$ increases. The important point is this: the sequence of essential bifurcations giving rise to a given point in $\Lambda_{\mu}$ is independent of the particular family chosen. This means that there are severe topological restrictions on the generation of periodic orbits by mappings like the logistic function.

The possibility of such a result is suggested by a remarkable result of Guckenheimer [2] which shows that the order in which the above bifurcations occur is independent of the family of mappings. The present paper extends these results in that we identify not only when but also where and how a particular periodic orbit appears. Viewed differently, we are able to trace the complete "family tree" of a given family of periodic orbits-which higher period points bifurcate away from it, and where these higher period families end up in $\Lambda_{\mu}$.

We finally remark that the results below are not completely new. They can be deduced, for example, from the work of Jonker [5]. Also, they are similar in spirit to results of Milnor and Thurston [6], and also to more recent work of Guckenheimer [4]. Our approach, however, is different, as we use only the topological properties of the global bifurcation diagram rather than symbolic dynamics to obtain our results.

1. Preliminaries. We consider smooth (at least $C^{3}$ ) one parameter families of maps $f_{\mu}: \mathbf{R} \rightarrow \mathbf{R}$ of the real line where the parameter $\mu \in[0, \infty)$. Throughout this paper, we assume that the family satisfies

(1) $f_{0}(x)=0$.

(2) $f_{\mu}(0)=f_{\mu}(1)=0$.

(3) For $\mu>0, f_{\mu}$ has a single critical point $c_{\mu}$ at which $f_{\mu}$ assumes its maximum.

(4) There is $b$ such that $\mu \geqslant b$ implies

$$
\left|f_{\mu}^{\prime}(x)\right|>1 \text { if } x, f_{\mu}(x) \in I=[0,1] \text {. }
$$

Clearly, the logistic function $f_{\mu}(x)=\mu x(1-x)$ is one such family.

The sequence of points $f_{\mu}^{n}(x)=f \circ f \circ \cdots \circ f(x)$ is called the orbit of $x$. A glance at the graph of $f_{\mu}$ shows that if $x \in I$ but $f_{\mu}(x) \notin I$, then $f_{\mu}^{n}(x) \notin I$ for $n \geqslant 2$. So any point which leaves $I$ under some iterate of $f_{\mu}$ remains outside of $I$ for all future iterates. In the sequel we shall restrict attention to points in $I$. The points whose entire orbits lie in $I$ thus form a compact invariant set which we denote by $\Lambda_{\mu}$. One of our goals is to understand how $\Lambda_{\mu}$ changes as $\mu$ increases.

Of prime importance in $\Lambda_{\mu}$ are the periodic orbits. These are points whose iterates satisfy $f_{\mu}^{n+i}(x)=f_{\mu}^{i}(x)$ for all $i>0$. The minimal number $n>0$ for which this equation holds is called the prime period of the orbit. It can be shown that for small $\mu>0, \Lambda_{\mu}$ consists of a finite number of periodic orbits. On the other hand, 
when $\mu \geqslant b$, it is easily seen that $\Lambda_{\mu}$ is a Cantor set and that there are exactly $2^{n}$ points in $\Lambda_{\mu}$ which lie on orbits of (not necessarily prime) period $n$. Denote by $\operatorname{Per}_{n}\left(f_{\mu}\right)$ the set of points of period $n$ for $f_{n}$. Equivalently, $\operatorname{Per}_{n}\left(f_{\mu}\right)$ consists of all points fixed by $f_{\mu}^{n}$. For polynomial mappings, $\operatorname{Per}_{n}\left(f_{\mu}\right)$ consists of a finite number of points. In general, however, this need not be the case. However, we shall place generic hypotheses on the family under consideration which will guarantee that $\operatorname{Per}_{n}\left(f_{\mu}\right)$ is finite.

As the parameter $\mu$ increases, the number of points in $\operatorname{Per}_{n}\left(f_{\mu}\right)$ changes at the bifurcation points. For generic families, these bifurcations are well understood. Either there is a saddle node bifurcation at which a new periodic point appears and then immediately splits into two periodic orbits of the same period, or else a flip bifurcation occurs and a new family of periodic points of twice the period bifurcates away from the given family. Both of these bifurcations can occur in reverse, i.e. with periodic points coalescing and/or disappearing. These generic bifurcations are well understood; we refer to [2] for more details and complete proofs. Henceforth we will assume that the given family $f_{\mu}$ is generic in the sense that at all points $x$ in $\operatorname{Per}_{n}\left(f_{\mu}\right)$, either the derivative $\left|\left(f_{\mu}^{n}\right)^{\prime}(x)\right| \neq 1$, or else there is a generic bifurcation in one of the above senses at $x$. All of our results below will be independent of the particular generic family, and so, at the end of the paper, we will be able to extend these results to arbitrary families satisfying hypotheses (1)-(4).

We now recall some basic facts about the symbolic dynamics of maps of the interval. Most of what follows is similar to results in Milnor and Thurston [6]. Let $z_{0}, z_{1}$ be the two preimages of 1 for fixed $\mu \geqslant b$. Suppose $z_{0}<z_{1}$ and let $I_{0}=\left[0, z_{0}\right]$ and $I_{1}=\left[z_{1}, 1\right]$. Then $f_{\mu}$ stretches both $I_{0}$ and $I_{1}$ homeomorphically over $I ; f_{\mu}$ increases on $I_{0}$ and decreases on $I_{1}$ and for each $x \in I_{0} \cup I_{1},\left|f_{\mu}^{\prime}(x)\right|>1$. Hence $\Lambda_{\mu}$ is a hyperbolic set for $\mu \geqslant b$.

For each $x \in \Lambda_{\mu}$, one may define a coding $h(x)=s_{0} s_{1} s_{2} \cdots$ where $s_{i}=0$ or 1 for each $i$. The sequence $h(x)$ is determined by $s_{i}=j$ if $f_{\mu}^{i}(x) \in I_{j}$. So $h(x)$ specifies which of the two intervals $I_{0}$ and $I_{1}$ the successive iterates of $x$ fall into.

Let $\Sigma$ denote the set of all one-sided sequences of 0's and 1's with the usual topology. $\Sigma$ is clearly homeomorphic to the Cantor middle thirds set. We define the shift automorphism $\sigma: \Sigma \rightarrow \Sigma$ by $\sigma\left(s_{0} s_{1} s_{2} \cdots\right)=s_{1} s_{2} s_{3} \cdots$. So $\sigma$ forgets the first term in a sequence and is thus a two-to-one mapping onto $\Sigma$. In the usual topology, $\sigma$ is also continuous. Moreover, the following proposition is well known:

Proposition 4. (1) $h: \Lambda_{\mu} \rightarrow \Sigma$ is a homeomorphism.

(2) $h \circ f_{\mu}=\sigma \circ h$, i.e. $h$ is a topological conjugacy between $f_{\mu} \mid \Lambda_{\mu}$ and $\sigma$.

(3) Periodic points of period $n+1$ for $f_{\mu}$ are in one-to-one correspondence via $h$ with periodic sequences of the form $s_{0} s_{1} \cdots s_{n} s_{0} \cdots s_{n} \cdots$.

Smale first proved these facts for the "horseshoe" diffeomorphism of the plane [9]. More detailed treatments of this example can be found in [7] or [8].

In this paper, we shall be concerned only with the periodic orbits. To simplify notation, we shall drop the tail of the infinite sequence of 0 's and 1's and consider a 
string of symbols $\underline{s}=s_{1} \cdots s_{n}$ as representing the periodic orbits $s_{1} \cdots s_{n} s_{1} \cdots s_{n} \cdots$. Moreover, we shall freely use the identification of $\operatorname{Per}_{n}(f)$ with such strings given by the topological conjugacy $h$. Let $\Sigma_{n}$ denote the set of all strings of length $n$ of the form $s_{1} \cdots s_{n}$.

For each $\mu \geqslant b$, the $2^{n}$ periodic points of period $n$ for $f_{\mu}$ are ordered in $I=[0,1]$ from left to right. This ordering remains unchanged as $\mu$ varies in the following sense. For $\mu \geqslant b$, there are $2^{n}$ smooth nonintersecting curves in $I \times[b, \infty)$ of the form $(x(\mu), \mu)$ satisfying $f_{\mu}^{n}(x(\mu))=x(\mu)$. Hence the ordering of the periodic points when $\mu=b$ determines the ordering for all $\mu>b$. As a result we need only consider changes in the periodic point structure of $f_{\mu}$ for $0<\mu<b$.

Number the points in $\operatorname{Per}_{n}\left(f_{b}\right) \cap I$ from left to right beginning at 0 and ending at $2^{n}-1$. Let $N(x)$ be the integer corresponding to $x$ in this ordering. Also, let $B(x)$ denote $N(x)$ in binary notation. The following proposition relates $N(x)$ to an ordering of strings of symbols of length $n$ in $\Sigma_{n}$.

Proposition 5. Let $h(x)=s_{1} \cdots s_{n}$. Then $B(x)=a_{1} \cdots a_{n}$ where $a_{j}=$ $\sum_{i=1}^{j} s_{i} \bmod 2$.

Proof. First recall that $s_{1} \cdots s_{n}$ is the unique point in $\operatorname{Per}_{n}\left(f_{b}\right)$ lying in $I_{s_{1}} \cdots s_{n}=I_{s_{1}} \cap f_{b}^{-1}\left(I_{s_{2}}\right) \cap \cdots \cap f_{b}^{-(n-1)}\left(I_{s_{n}}\right)$ and that $f_{b}^{n}$ is monotonic on this closed interval. Furthermore,

$$
\begin{aligned}
I_{s_{0} \cdots s_{n}} & =I_{s_{0}} \cap f_{b}^{-1}\left(I_{s_{1}}\right) \cap \cdots \cap f_{b}^{-n}\left(I_{s_{n}}\right) \\
& =I_{s_{0}} \cap f_{b}^{-1}\left(I_{s_{1}} \cap \cdots \cap f_{b}^{-(n-1)}\left(I_{s_{n}}\right)\right) \\
& =I_{s_{0}} \cap f_{b}^{-1}\left(I_{s_{1}} \cdots s_{n}\right) .
\end{aligned}
$$

This means that the ordering of $\operatorname{Per}_{n+1}\left(f_{b}\right)$ is obtained from the ordering $\operatorname{Per}_{n}\left(f_{b}\right)$ as follows. Since $f_{b}$ increases on $I_{0}$, we first list all strings of length $n$ in order but preceded by a 0 . Then $f_{b}$ decreases on $I_{1}$ so that the strings of length $n$ are then listed in reverse order and preceded by a 1 . This new list is the ordering of $\operatorname{Per}_{n+1}\left(f_{b}\right)$.

Now by induction, we first note that $B(0)=0$ and $B(1)=1$ so that $B$ gives the binary location for the fixed points of $f_{b}$. Now assume that $a_{1} \cdots a_{n}=$ $B\left(s_{1} \cdots s_{n}\right)$ gives the binary location of $s_{1} \cdots s_{n} \in \Sigma_{n}$. Then the above remarks show that $B\left(0, s_{1} \cdots s_{n}\right)=0 a_{1} \cdots a_{n}$ and $B\left(1, s_{1} \cdots s_{n}\right)=1 \hat{a}_{1} \cdots \hat{a}_{n}$ where $\hat{a}_{j}=a_{j}+1 \bmod 2$. Since $a_{1} \cdots a_{n}+\hat{a}_{1} \cdots \hat{a}_{n}=1 \cdots 1$ it follows that

$$
B\left(0, s_{1}, \ldots, s_{n}\right)<B\left(0, t_{1} \cdots t_{n}\right), \quad B\left(1, s_{1} \cdots s_{n}\right)>B\left(1, t_{1} \cdots t_{n}\right),
$$

iff $B\left(s_{1} \cdots s_{n}\right)<B\left(t_{1} \cdots t_{n}\right)$. Consequently $B\left(s_{0} s_{1} \cdots s_{n}\right)$ gives the binary ordering of $\operatorname{Per}_{n+1}\left(f_{b}\right)$ as required.

The preceding proposition relates a geometric property of $f_{b}$-the ordering of $\operatorname{Per}_{n}\left(f_{b}\right)$ in $I$-with an algebraic property of strings of length $n$ in $\Sigma_{n}$-the binary location. We now discuss a second such link between the geometry of $f_{b}$ and the algebra of $\sigma$. For an isolated periodic point $p \in \operatorname{Per}_{n}\left(f_{\mu}\right)$, we let $\operatorname{Ind}_{n}(p)$ denote the topological intersection number of $\operatorname{graph}\left(f_{\mu}^{n}\right)$ with the diagonal in $I \times I$ at $(p, p)$. That is, $\operatorname{Ind}_{n}(p)=+1$ if $f_{\mu}^{n}(x)-x$ is increasing at $p$; $\operatorname{Ind}_{n}(p)=-1$ if $f_{\mu}^{n}(x)-x$ is 
decreasing at $p$; and $\operatorname{Ind}_{n}(p)=0$ otherwise. For a generic family of mappings, all periodic points are isolated, and so $\operatorname{Ind}_{n}(x)$ is well defined for each $x$ in $\operatorname{Per}_{n}\left(f_{\mu}\right)$. Also, if $f_{\mu}$ is a polynomial of degree $\geqslant 2$, then again each periodic point is isolated and the index is well defined.

For strings of length $n$ in $\Sigma_{n}$ we also define an algebraic index. Let

$$
I\left(s_{1} \cdots s_{n}\right)=(-1)^{\sum_{i-1}^{n} s_{i}} \text {. }
$$

$I$ is related to the topological index in the natural way.

Proposition 6. Let $x \in \operatorname{Per}_{n}\left(f_{b}\right)$. Then $I(h(x))=\operatorname{Ind}_{n}(x)$.

Proof. Suppose $h(x)=s_{1} \cdots s_{n}$ and that $B\left(s_{1} \cdots s_{n}\right)=a_{1} \cdots a_{n}$. Then $I\left(s_{1} \cdots s_{n}\right)=(-1)^{\Sigma s_{j}}=(-1)^{a_{n}}$. On the other hand, for fixed points of $f_{b}^{n}$, the derivative $\lambda(x)=\left(f_{b}^{n}\right)^{\prime}(x)$ is alternately positive and negative with $|\lambda(x)|>1$. Since $\lambda(0)>1$ by assumption, it follows that

$$
\operatorname{Ind}_{n}(x)=(-1)^{N(x)} \text {. }
$$

Then the result is an immediate consequence of the previous proposition, as $N(x)$ is even or odd depending on whether $a_{n}=0$ or $a_{n}=1$.

2. Families of periodic orbits. In this section we complete the description of the generation of periodic orbits as $\mu$ increases. The generic hypotheses on $f_{\mu}$ imply that the set of solutions of the equation $f_{\mu}^{n}(x)=x$ forms a union of smooth curves in $I \times[0, b]$ which we denote by $\Gamma_{n}$. The associated picture in $I \times[0, b]$ is called the global bifurcation diagram of period $n$.

Certain of the curves in $\Gamma_{n}$ extend all of the way to the line $\mu=b$, while others do not. Our goal in this section is to identify which of the periodic points for $\mu=b$ are related via sequences of saddle node and/or flip bifurcations to other points in $\operatorname{Per}_{n}\left(f_{b}\right)$.

Toward that end, for $p \in \operatorname{Per}_{n}\left(f_{b}\right)$, we denote by $\gamma(p)$ the smooth curve in $\Gamma_{n}$ which terminates at $(p, b) \in I \times[0, b]$. This curve is called the fixed point curve associated to $p$. If $p$ is not a fixed point for $f_{b}$, then $\gamma(p)$ rejoins $\mu=b$ at a second point $q$. That is, $\gamma(p)=\gamma(q)$. We say that $p$ is related to $q$ in this case and write $p \sim q$. Note that $q$ may or may not be on the $f_{b}$-orbit of $p$. Indeed, the period 2 points associated to the sequences 01 and 10 in $\Sigma_{2}$ are related and do lie on the same orbit, while the period 3 orbits with sequences 101 and 100 are related, but necessarily lie on distinct orbits. This follows immediately from the considerations below.

If $p$ and $q$ both have prime period $n$, then it follows from our generic hypotheses that either $p$ is related to $q$ or else $\gamma(p) \cap \gamma(q)=\varnothing$. For the only way two distinct curves in $\Gamma_{n}$ can cross is at a flip bifurcation. Here, either a new orbit with twice the period bifurcates away, or else such an orbit coalesces with the original family. On the other hand, if $p$ and/or $q$ have period less than $n$, then $\gamma(p)$ may meet $\gamma(q)$ at a point $(y, \nu)$ where a flip bifurcation occurs. In this case, the prime period of one of the points, say $q$, must be exactly twice the prime period of the other. We say that $p$ gives birth to $q$ and denote this by $p \rightarrow q$. More generally, we say that $q$ is 
a descendant of order $k$ of $p$ if there is a sequence $q_{0}=p, q_{1}, \ldots, q_{k}=q$ of points in $\operatorname{Per}_{n}\left(f_{b}\right)$ satisfying $q_{i} \rightarrow q_{i+1}$ for $i=0,1, \ldots, k-1$. We write $p \stackrel{k}{\rightarrow} q$ in this case. Note that if $p \stackrel{k}{\rightarrow} q$, then the prime period of $q$ is $2^{k}$ times the prime period of $p$. Also if $p$ gives birth to $q$, then $q$ is a descendant of $p$ of order 1 .

Many periodic points for $f_{b}$ are not descendants of points with lower periods; that is, many periodic points do not arise out of flip bifurcations. A point $p$ in $\operatorname{Per}_{n}\left(f_{b}\right)$ is called primary if each point in $\gamma(p)$ has the same prime period. Equivalently, $p$ is a primary periodic point provided there are no points $(y, \nu)$ along $\gamma(p)$ which also lie on $\gamma(q)$ for some $q$ with lower period than $p$. Primary periodic points are clearly those which "begin" at saddle nodes.

REMARK. Note that fixed point curves which begin at flip bifurcations may also admit saddle node bifurcations even before the parameter value corresponding to the flip bifurcations. See Figure 1 .

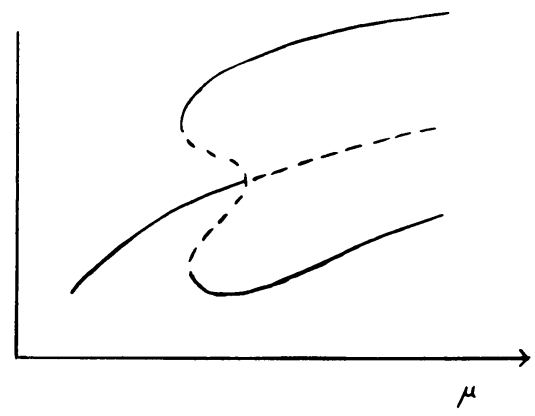

FIGURE 1. An "inverse flip" bifurcation

We define the period of a fixed point curve $\gamma(p)$ to be the prime period of $p$. The following proposition shows that there is at most one point along $\gamma(p)$ which has prime period less than $p$.

Proposition 7. Suppose $\gamma(p)$ has period $n$. Then there exists at most one point $(y, \nu) \in \gamma(p)$ with $f_{\mu}^{m}(y)=y$ with $m<n$.

Proof. Let $\left(y_{i}, \nu_{i}\right)$ be two such points with $i=1,2$ and let $\gamma_{1}$ denote that portion of $\gamma(p)$ connecting $\left(y_{1}, \nu_{1}\right)$ to $\left(y_{2}, \nu_{2}\right)$. We may assume that the prime period of any point $(x, \mu) \in \gamma_{1}$ is $n$. By our generic hypotheses, $\left(y_{i}, \nu_{i}\right)$ are not endpoints of $\gamma_{1}$, and both $y_{1}$ and $y_{2}$ have period $n / 2$. Let $\gamma_{2}$ denote the curve $\left(f_{\mu}^{n / 2}(x), \mu\right)$ for each $(x, \mu) \in \gamma_{1}$. Then $\gamma_{1}$ and $\gamma_{2}$ are smooth curves which join together smoothly at $\left(y_{1}, \nu_{1}\right)$ and $\left(y_{2}, \nu_{2}\right)$. This implies that $\gamma_{1} \cup \gamma_{2}$ is a smooth closed curve in $\Gamma_{n}$. Hence $\gamma_{1} \cup \gamma_{2}$ cannot meet $\mu=b$ and, in particular, $(p, b) \notin \gamma_{1} \cup \gamma_{2}$. This gives a contradiction and completes the proof.

Our goal now is to identify which periodic points give birth to higher period points. In the next section we shall deal with the converse: which periodic points in $\operatorname{Per}_{n}\left(f_{b}\right)$ are primary and which arise out of flip bifurcations. This will allow us to give a complete "family history" of any point in $\operatorname{Per}_{n}\left(f_{b}\right)$. The important point is that these relationships turn out to be independent of the particular generic family chosen. Hence there are severe topological restrictions on how periodic points can 
arise and persist in such a family. These ideas were suggested to us by a remarkable result of Guckenheimer which we now discuss.

For $p \in \operatorname{Per}_{n}\left(f_{b}\right)$, let $m(p)$ denote the minimum $\mu$-value along the associated fixed point curve $\gamma(p)$ in $I \times[0, b]$. Also, denote by $M(p)$ the point along the orbit of $p$ with largest $x$-coordinate. In our terminology, Guckenheimer's result may then be stated as follows:

TheOREM (GUCKENHEIMER [2]). Suppose $f_{\mu}$ is a generic family of mappings. Let $p_{1}$, $p_{2} \in \operatorname{Per}_{n}\left(f_{b}\right)$ and suppose $\operatorname{Ind}_{n}\left(p_{i}\right)=-1$. Then $m\left(p_{1}\right)<m\left(p_{2}\right)$ iff $M\left(p_{1}\right)<M\left(p_{2}\right)$.

Using this theorem one can decide the order in which bifurcations must occur; that is, given a pair of periodic points, one can determine which of the two was created by an earlier bifurcation. Our goal in this paper is to extend these results by identifying the complete bifurcation history of each point in $\operatorname{Per}_{n}\left(f_{b}\right)$.

As above, we continue to identify points $\operatorname{in}^{\operatorname{Per}_{n}}\left(f_{b}\right)$ with their associated strings of length $n$ in $\Sigma_{n}$. For $s=s_{1} \cdots s_{n} \in \Sigma_{n}$, we define a new sequence $\underline{\hat{s}}=$ $s_{1} \cdots s_{n-1} \hat{s}_{n}$, where $\hat{s}_{n}=s_{n}+1 \bmod 2$. The sequence $\underline{\hat{s}}$ is said to be conjugate to $\underline{s}$. Our main tool in the sequel is the following theorem.

THEOREM A. Let $\underline{s}=s_{1} \cdots s_{n} \in \Sigma_{n}$ satisfy

(1) $s$ has prime period $n$.

(2) $I(s)=-1$.

(3) $M(s)=s$.

Then there is a unique fixed point curve with period $2 n$ which intersects both $\gamma(s)$ and the line $\mu=b$. The rightmost point on this orbit is conjugate to the sequence $s_{1} \cdots s_{n} s_{1} \cdots s_{n}$, i.e. is given by $s_{1} \cdots s_{n} s_{1} \cdots \hat{s}_{n}$.

REMARK. The point on $\gamma(s)$ at which the higher period fixed point curve branches off is called its birthplace. Note that, as in Figure 1, it is possible for a given family of periodic orbits to "live" before its birthplace. Also, if $\underline{s}=s_{1} \cdots s_{n}$ and $\underline{t}=$ $t_{1} \cdots t_{k}$, we denote by $s t$ the new string of length $n+k$ given by $s_{1} \cdots s_{n} t_{1} \cdots t_{k}$.

PROof. Let $\lambda_{n}(y)$ denote the derivative $f_{\mu}^{n}(y)$. Let $\xi$ be the portion of the curve $\gamma(s)$ connecting $(s, b)$ and the point $(y, m(s))$, i.e. the point with minimal $\mu$-period along $\gamma(s)$. At $y$, we have $\lambda_{n}(y)=1$, whereas $\lambda_{n}(s)<-1$. Hence there are an odd number of isolated points along $\xi$ at which $\lambda=-1$. At each of these points, a family of $2 n$ periodic points bifurcates away from $\xi$. We claim that exactly one of these families persists up to $\mu=b$. To see this, we first observe that an odd number of fixed point curves must persist up to $\mu=b$. This follows from Proposition 7 which shows that the fixed point curves branching away from $\gamma(s)$ can only disappear in pairs. Now let $\underline{s}^{1}, \ldots, \underline{s}^{2 k+1}$ denote the set of points in $\operatorname{Per}_{2 n}\left(f_{b}\right)$ satisfying $B\left(\underline{s}^{j}\right)>B(s)$ and $\gamma\left(\underline{s}^{j}\right) \cap \gamma(s) \neq \varnothing$. We may assume that $B\left(\underline{s}^{i}\right)<B\left(\underline{s}^{j}\right)$ if $i<j$. Then if $k>0$, we must have that $I\left(s^{1}\right)=I\left(s^{3}\right)=-1$, since there must be an even number of points in $\operatorname{Per}_{2 n}\left(f_{b}\right)$ between $s^{i}$ and $s^{i+1}$ and since $I(s s)=1$. Since $M(s)=s$, it also follows that $M\left(\underline{s}^{1}\right)=\underline{s}^{1}$ and $M\left(s^{3}\right)=\underline{s}^{3}$, for otherwise some of the fixed point curves associated to the points on the orbit of $s^{i}$ would intersect 
each other. By Guckenheimer's theorem, it then follows that $m\left(s^{3}\right)>m\left(s^{1}\right)$. But this implies that the curves $\gamma\left(\underline{s}_{-}^{1}\right)$ and $\gamma\left(s^{3}\right)$ must cross. Since both curves have period $2 n$, this is impossible.

We now show that this unique bifurcation family meets $\mu=b$ at $s \hat{s}$. First observe that, by Proposition $6, I(s s)=1$. Hence $B(s s)=a_{1} \cdots a_{2 n}$ with $\bar{a}_{2 n}=0$. Then $B(s \hat{s})=a_{1} \cdots a_{2 n-1} \hat{a}_{2 n}$ with $\hat{a}_{2 n}=1$ so that $\overline{B(s \hat{s})}>B(s s)$. If the bifurcation family meets $\mu=b$ at some larger symbol, say $\underline{t}$, then we must have $m(t)<m(s \hat{s})$ since $\gamma(s \hat{s})$ has period $2 n$ and cannot cross $\gamma(\underline{t})$. Again invoking Guckenheimer's result, it follows that there must be a larger point than $s \hat{s}$ on its orbit. We now show that in fact $s \hat{s}$ is the largest point on its orbit, and this contradiction will finish the proof.

Since $B(\underline{s s})<B(\underline{s \hat{s}})$ and $B(s)>B\left(\sigma^{k} s\right)$ for $k=1, \ldots, n-1$, it follows that

$$
B\left(\sigma^{k} \underline{s}, \sigma^{k} \hat{s}\right)<B\left(\sigma^{k} \underline{s}, \sigma^{k} \underline{s}\right)<B(\underline{s s})
$$

for $k=1, \ldots, n-1$. Hence we need only show that $B\left(\sigma^{k} \hat{s}, \sigma^{k} s\right)<B(s s)$ for $k=1, \ldots, n$ to complete the proof. We show instead that $B\left(\sigma^{k} \underline{s}\right)<B \overline{(s)}$ for $k=0, \ldots, n-1$.

Let $B(s)=b_{1} \cdots b_{n}$. Since $I(s)=-1$, it follows that $b_{n}=1$ so that when $k=0$, $B(\underline{\hat{s}})=b_{1} \cdots b_{n-1} \hat{b}_{n}<b_{1} \cdots b_{n}=B(s)$.

For $k=1, \ldots, n-1$, we argue as follows. Suppose $B\left(\sigma^{k} \hat{s}\right)>B(s)$. Let $B\left(\sigma^{k} s\right)$ $=a_{1} \cdots a_{n}$. Then $B\left(\sigma^{k} \underline{s}\right)=a_{1} \cdots a_{n-k-1} \hat{a}_{n-k} \cdots \hat{a}_{n}$. Since $B\left(\sigma^{k} s\right)<B(s)$ it follows that $a_{1}=b_{1}, \ldots, a_{n-k-1}=b_{n-k-1}$. Since $B\left(\sigma^{k} \hat{s}\right)>B(s)>B\left(\sigma^{k} s\right)$ we must then have $a_{n-k}=0$ and $\hat{a}_{n-k}=1$. Thus there are two cases: either $b_{n-k}=0$ or $b_{n-k}=1$.

Suppose first that $b_{n-k}=0$. Since $s_{1}=1$ it follows that $a_{n-k-1}=1$ and that $a_{n-k-1} \cdots a_{n}<b_{n-k-1} \cdots b_{n}$. But we have

$$
a_{n-k+1} \cdots a_{n}=B\left(s_{1} \cdots s_{k}\right) \geqslant B\left(s_{n-k+1} \cdots s_{n}\right)=b_{n-k+1} \cdots b_{n} \text {. }
$$

This contradiction establishes the result when $b_{n-k}=0$. If $b_{n-k}=1$ we must have $b_{n-k+1} \cdots b_{n}<\hat{a}_{n-k+1} \cdots \hat{a}_{n}$. Since $s_{1}=1$, we have $\hat{a}_{n-k+1}=0$ and therefore $b_{n-k+1}=0$. Since $b_{n-k}=1$, it follows that $s_{n-k+1}=1$. Hence $B\left(s_{n-k+1} \cdots s_{n}\right)=$ $b_{n-k+1} \cdots b_{n}$. As before, $B\left(s_{1} \cdots s_{k}\right)=\hat{a}_{n-k+1} \cdots \hat{a}_{n}$ so that

$$
a_{n-k+1} \cdots a_{n}=B\left(s_{1} \cdots s_{k}\right) \geqslant B\left(s_{n-k+1} \cdots s_{n}\right)=\hat{b}_{n-k+1} \cdots \hat{b}_{n} .
$$

Therefore $\hat{a}_{n-1+1} \cdots \hat{a}_{n}<b_{n-k+1} \cdots b_{n}$ and again we have a contradiction.

As a consequence of Theorem A, we can determine recursively the higher period orbits which are descendants of order $k$ of a given periodic point. For example, we have

COROllary B. Let $p$ correspond to the fixed point of $f_{b}$ with negative index. Then $p \stackrel{k}{\rightarrow} \underline{s}^{k}$ where $M\left(\underline{s}^{k}\right)=\underline{s}^{k}$ and the $\underline{s}^{k}$ are determined recursively by the formula $\underline{s}^{k+1}=\underline{s}^{k} \underline{s}^{k}$.

In particular, the first few periodic points related to $p$ are given by the sequences $1,10,1011,10111010,1011101010111011 \ldots$ A similar scheme holds for any other periodic point with negative index. 
3. Related periodic orbits. Our goal in this section is to describe the past history of a given periodic point in $\Sigma_{n}$. More precisely, we present an algorithm which decides whether two sequences are related, or more generally, if one is a descendant of order $k$ of the other.

Let $\underline{s}=s_{1} \cdots s_{n}$ and $\underline{t}=t_{1} \cdots t_{n}$ and suppose $\underline{s} \sim \underline{t}$. We say that $\underline{s}$ and $\underline{t}$ separate the critical point if $s_{1} \neq t_{1}$. In this case, the $x$-coordinates of the two ends of $\gamma(s)$ lie on opposite sides of the critical point of $f_{b}$.

LeMma 1. Suppose $\underline{s} \sim \underline{t}$ and $\underline{s}$ and $\underline{t}$ separate the critical point. Then $\sigma^{k} \underline{s}$ and $\sigma^{k} \underline{t}$ do not separate the critical point unless $\sigma^{k} \underline{\underline{s}}=\underline{s}$ or $\sigma^{k} \underline{\underline{s}}=\underline{t}$.

Proof. If $\sigma^{k} \underline{s}$ and $\sigma^{k} \underline{t}$ separate the critical point, then $\gamma(s)$ intersects $\gamma\left(\sigma^{k} s\right)$ since $m(s)=m\left(\sigma^{k} s\right)$. Since $\sigma^{k} \underline{s}$ and $\underline{s}$ have the same period, it follows that $\gamma(s)=\gamma\left(\sigma^{k} s\right)$.

LemMA 2. Suppose $M(s)=\underline{s}$ and $\underline{s} \sim \underline{t}$. Then $\sigma^{-1}(s)$ and $\sigma^{-1}(\underline{t})$ separate the critical point.

Proof. There is one point on the orbits of $\underline{s}$ and $\underline{t}$ which separates the critical point, say $s_{0}$ and $\underline{t}_{0}$. Consider $\sigma\left(s_{0}\right)$ and $\sigma\left(\underline{t}_{0}\right)$. If $B(s)>B\left(\sigma s_{0}\right)$ then $B(t)>B\left(\sigma \underline{t}_{0}\right)$ also, for otherwise $\gamma(s)=\gamma\left(\sigma s_{0}\right)$. Now the graph of $f_{b}$ shows that $\sigma^{-1} \underline{s}$ and $\sigma^{-1} t$ are contained between $s_{0}$ and $\underline{t}_{0}$. Hence it follows that $\gamma\left(s_{0}\right)$ and $\gamma\left(\sigma^{-1} s\right)$ must intersect.

We can now describe when two sequences are related.

THEOREM C. Suppose $\underline{s} \sim \underline{t}$ and $M(s)=\underline{s}$. Then either

(a) $\underline{t}=\hat{s}$, or

(b) $\underline{s}=\underline{u \hat{u}}$ and $t=\underline{\hat{u} u}$.

Conversely, if $M(s)=\underline{s}$ and $\underline{s}=\underline{u \hat{u}}$, then $\underline{s} \sim \underline{\hat{u} u}$. Otherwise, $\underline{s} \sim \hat{\underline{s}}$.

Proof. Suppose first that $\underline{s} \sim t$ with $M(s)=\underline{s}$. Let $s_{0}$ and $\underline{t}_{0}$ be the unique points on the orbits of $\underline{s}$ and $\underline{t}$ which separate the critical point. We have $s_{0}=s_{1} \cdots s_{k}$ and $\underline{t}_{0}=\hat{s}_{1} s_{2} \cdots s_{k}$. Now either $s_{0}$ and $\underline{t}_{0}$ lie on the same orbit or else they lie on distinct orbits. In the first case it follows that $\underline{t}_{0}=\sigma^{m} s_{0}$ where $m=k / 2$, so that $\underline{s}_{0}=s_{1} \cdots s_{m} \hat{s}_{1} \cdots s_{m}$ and $\underline{t}_{0}=\hat{s}_{1} \cdots s_{m} s_{1} \cdots s_{m}$. By Lemma $2, \underline{s}=\sigma s_{0}$ so that $\underline{s}=\underline{u \hat{u}}$ as required. In the second case we must have $\underline{s}=s_{2} \cdots s_{k} s_{1}$ and $\underline{t}=$ $s_{2} \cdots s_{k} \hat{s}_{1}$ again as required.

For the converse, we first assume that $M(s)=\underline{s}$ and that $\underline{s}=u \hat{u}$ where $\underline{u}=$ $s_{1} \cdots s_{n}$. We first claim that $I(\underline{u})=-1$. Since $M(s)^{-}=\underline{s}$, we have $\overline{B(u \hat{u})}>B(\overline{\hat{u}} u)$. Let $B(u)=a_{1} \cdots a_{n}$. Then we have

$$
a_{1} \cdots a_{n} \geqslant B(\underline{\hat{u}}) a_{1} \cdots a_{n-1} \hat{a}_{n} .
$$

It follows that $a_{n}=1$. Hence $\sum_{j=1}^{n} s_{j}=1 \bmod 2$, and we have that $I(u)=-1$. We also clearly have $B\left(\sigma^{k} u\right)<B(\underline{u})$ for $k=1, \ldots, n-1$, so that by Theorem $A, \underline{u}$ gives birth to $\underline{s}=\underline{u \hat{u}}$. Since $\sigma^{n} \underline{u \hat{u}}=\hat{u} u$, it follows that $\underline{u \hat{u}} \sim \hat{u} u$.

On the other hand, if $\underline{s}$ is not of the form $\underline{u \hat{u}}$ and $\underline{\underline{s}} \sim \bar{t}$, then by Lemma $2, \sigma^{-1} \underline{s}$ and $\sigma^{-1} \underline{t}$ separate the critical point. Hence $\underline{t}=\overline{\hat{s}}$. This completes the proof. 
Thus, to determine how a given orbit arises, we iterate $\sigma$ until we find $M(s)$. If $M(s)=u \hat{u}$, then the orbit originates at a flip bifurcation along the orbit of $\underline{u}$. In all other cases, $\underline{s}$ arises as a saddle node, and in fact if $M(s)=\underline{v}$, then $\underline{v} \sim \hat{v}$. Using this procedure, one can construct the entire prior history of a given periodic orbit.

Finally, we remark that our results above go over immediately to the case of a family of polynomial mappings satisfying hypotheses (1)-(4). Let $f_{\mu}$ be such a family. Now $f_{\mu}$ does not in general meet the generic hypotheses imposed in $\$ 2$. However, the global bifurcation diagram of period $n$ does consist of a finite number of continuous curves, since these curves are given as the zero set of a polynomial in two variables.

Let $f_{\mu}^{i}$ be a sequence of mappings converging to $f_{\mu}$ in the $C^{1}$ topology (on $I$ ). For each $i$, there is a number $b_{i}$ such that $f_{\mu}^{i} \mid \Lambda_{i}$ is topologically conjugate to the shift on two symbols for $\mu \geqslant b_{i}$. Let $\underline{s} \in \Sigma_{n}$ and let $\gamma_{i}(s)$ be the fixed point curve associated to $\underline{s}$ for $f_{\mu}^{i}$ in $I \times\left[0, b_{i}\right]$. By Theorem A, there is $\underline{t} \in \Sigma_{n}$ such that $\underline{t} \in \gamma_{i}(s)$ for all $i$. Since $f_{\mu}^{i} \rightarrow f_{\mu}$ uniformly on $I$, the curves $\gamma_{i}(s)$ converge to a closed set $G(s)$ which contains a continuous curve $\gamma(s)$ connecting $\underline{s}$ and $\underline{t}$ in $\Lambda_{i} \times\{b\}$. Hence $\underline{s} \sim \underline{t}$ for $f_{\mu}$ also. The other relationships among the periodic points follow similarly.

\section{REFERENCES}

1. R. Devaney and Z. Nitecki, Shift automorphisms in the Hénon mapping, Comm. Math. Phys. 67 (1979), 137-146.

2. J. Guckenheimer, On the bifurcation of maps of the interval, Invent. Math. 39 (1977), 165-178.

3. , The bifurcation of quadradic functions, Bifurcation Theory and Applications in Scientific Disciplines, Ann. N. Y. Acad. Sci. 316 (1979), 78-85.

4. _ Bifurcations of dynamical systems, Dynamical Systems, Birkhäuser, Boston, Mass., 1980, pp. 115-232.

5. L. Jonker, Periodic orbits and kneading invariants, Bull. London Math. Soc. (to appear).

6. J. Milnor and W. Thurston, On iterated maps of the interval. I, II (to appear).

7. J. Moser, Stable and random motions in dynamical systems, Ann. of Math. Studies, no. 77, Princeton Univ. Press, Princeton, N. J., 1973.

8. Z. Nitecki, Differentiablle dynamics, MIT Press, Cambridge, Mass., 1971.

9. S. Smale, Diffeomorphisms with many periodic points, Differential and Combinatorial Topology, Princeton Univ. Press, Princeton, N. J., 1965.

Department of Mathematics, Tufts University, Medford, MassachusetTs 02155

Current address: Department of Mathematics, 264 Bay State Road, Boston University, Boston, Massachusetts 02215 